\title{
MULTIPLE DISEASE RESISTANCE TO POWDERY MILDEW, BACTERIAL BLIGHT, AND ALTERNARIA BLIGHT IN LILACS (SYRINGA SPP.).
}

\author{
By Margaret T. Mmbaga1, Roger J. Sauvé ${ }^{\text {, Emmanuel Nnodu }}{ }^{3}$, and Suping Zhou ${ }^{4}$
}

\begin{abstract}
Fifty-six lilac accessions were evaluated in a 4-year study for resistance to powdery mildew caused by Microsphaera syringae, bacterial blight caused by Pseudomonas syringae pv. syringae, and Alternaria blight caused by Alternaria alternata. Accessions included 39 cultivars of Syringa vulgaris, four of S. prestoniae, three of $S$. hyacinthiflora, two of S. josiflexa, two of S. meyeri, two of $S$. reticulata, and one each of S. patula, S. chinensis, S. henryi, and $S$. microphylla. Of these, six accessions were resistant or moderately resistant to Alternaria blight and powdery mildew, four to bacterial blight and Alternaria blight, and 20 to powdery mildew and bacterial blight. Two cultivars of S. meyeri, 'Dwarf Korean' and 'Palibin', were resistant to all three pathogens. During this study, the most serious disease of lilacs in middle Tennessee, U.S., was Alternaria blight. It caused severe leaf scorching and defoliation that began in early July. Powdery mildew and bacterial blight caused mostly superficial damage with little effect on growth.

Key Words. Alternaria alternata; disease complex; Microsphaera syringae; plant health care; Pseudomonas syringae pv. syringae; Syringa spp.; variety selection.
\end{abstract}

Lilacs (Syringae spp.) have long been favorites landscape plants in North America. These ornamental shrubs and small trees, native of Asia and southeastern Europe, are easy to grow and can display showy, fragrant flowers and attractive foliage. Most are susceptible to powdery mildew caused by Microsphaera syringae (Sinclair et al. 1987). This disease is a nonlethal but aesthetic persistent problem in the southeastern region of the United States. It reduces the salability of lilacs due to the powdery appearance of mycelia, conidiophores, and conidia that develop on the surfaces of leaves and shoots. Although uncommon, severe infection can result in leaf necrosis, leaf distortion, chlorosis, premature leaf drop, and decreased growth (Clement et al. 1994). Although some resistant cultivars are available, they are not widely used (Hibben et al. 1977; Jones 1986; Clement et al. 1994; Widham et al. 1995).

Severe infection of Pseudomonas syringae pv. syringae, the causal agent of bacterial blight, can be a destructive disease in most Chinese, Japanese, Persian, and common lilacs (Sinclair et al. 1987). Bacterial blight commonly occurs during the early spring when the weather is cool and wet. Actively growing tissue is killed, resulting in the dieback of young shoots and flower clusters (Sinclair et al. 1987; Pschedt and Moorman 2001). Infected leaf petioles and succulent stems develop lesions, droop, wither, and turn brown. Maturing stems may remain erect and may develop brown to black streaks, while mature stems usually remain disease free.

During midsummer 1996, a leaf blight disease caused by Alternaria alternata was first observed in middle Tennessee (Mmbaga and Sheng 1997). Since then, it has increased in severity and now causes significant damage on many lilac cultivars (Mmbaga et al. 2003). Symptoms of this disease consist of brown necrotic lesions that often coalesce to form large blotches. Lesions may develop concentric rings with ash-colored center zones or remain irregular-shaped blotches without rings. Infected leaves of most cultivars evaluated suddenly wilted, died, and dropped off quickly. In others, a yellow halo developed around each lesion before wilting.

The objective of this study was to identify lilac taxa resistant to powdery mildew, bacterial blight, and Alternaria blight and to a disease complex involving a combination of these diseases.

\section{MATERIALS AND METHODS Germplasm Evaluation for Resistance to Multiple Diseases}

A total of 56 cultivars of nine species were evaluated. These included 39 cultivars of $S$. vulgaris, two of $S$. meyeri, one of $S$. patula, four of S. prestoniae, two of S. josiflexa, three of $S$. hyacinthiflora, two of $S$. reticulata, one of $S$. henryi, one of $S$. chinensis, and one of S. microphylla. Accessions were obtained from commercial nurseries. Plants were established in May 1994 at the Tennessee State University Nursery Crop Research Center in McMinnville, Tennessee, using 1.83 m (5.9 $\mathrm{ft}$ ) within-row plant spacing and $2.4 \mathrm{~m}(7.9 \mathrm{ft})$ between-row spacing. All plants were irrigated using drip irrigation whenever needed and fertilized during the first week of May of each growing season with a controlled-release fertilizer (14 $\mathrm{N}-14 \mathrm{P}-14 \mathrm{~K})$ at the rate of $41.7 \mathrm{~g} / \mathrm{m}^{2}\left(1.8 \mathrm{oz} / \mathrm{yd}^{2}\right)$. The experimental design consisted of a randomized complete block design with five replicates for each cultivar in which each cultivar was represented by one tree in each of the five rows. 


\section{Disease Development and Evaluation}

Previously infected plant parts and infested leaf debris provided the primary source of inoculum for the study. No additional inoculum was applied. Disease severity of bacterial and fungal plant pathogens was evaluated at monthly intervals. The study was initiated in early May and terminated in October of each growing season. The diseases rating of Horsfall-Barratt (1945) was used, with $1=1 \%$ to $10 \%, 2=11 \%$ to $25 \%, 3=26 \%$ to $50 \%, 4=51 \%$ to $75 \%$, and $5=76 \%$ to $100 \%$ of plant foliage showing signs or disease symptoms. For consistent readings, disease assessment was performed by the same research scientist throughout the experiment. Although several readings were taken during the growing season, readings taken in May targeted bacterial blight incidence. In August, powdery mildew and Alternaria blight incidence are reported. Both powdery mildew and Alternaria blight had the greatest visible impact in those months. During the study, a few cultivars in the collection were killed; thus, only cultivars that were rated during each season are reported in this article.

Each accession was evaluated for susceptibility based on mean disease readings collected during each growing season. Plants were categorized as resistant (R), moderately resistant (MR), moderately susceptible (MS), or susceptible (S), where 0 to 1.0 (R), 1.1 to 2.0 (MR), 2.1 to 2.9 (MS), and 3.0 to 5.0 (S). Due to variations in climactic condition among the seasons, the final rating used for each accession was the highest one obtained.

Because Alternaria blight was first observed in middle Tennessee during summer 1996, proof of pathogenicity tests were performed using Koch's rules (Koch 1882; Smith 1905). Once performed, 7-day-old cultures on potato dextrose agar (Barnett and Hunter 1998) were used to prepare $10^{6} \mathrm{spores} / \mathrm{mL}$ suspensions in distilled water to inoculate the four different taxa. Control plants were sprayed with distilled water. The following lilacs were assayed: S. prestoniae 'Isabella', S. prestoniae 'James McFarlane', S. meyeri 'Dwarf Korean', and S. vulgaris (unnamed common lilac).

Inoculated plants were incubated in a moist chamber at $28^{\circ} \mathrm{C}\left(82^{\circ} \mathrm{F}\right)$ and $100 \%$ relative humidity for 24 hours and subsequently moved to a greenhouse maintained at $25^{\circ} \mathrm{C} \pm$ $4^{\circ} \mathrm{C}\left(77^{\circ} \mathrm{F} \pm 7^{\circ} \mathrm{F}\right)$. Disease symptoms were recorded 10 weeks later. A randomized complete block design with four replications of single-tree per treatment was used.

\section{Statistical Analyses}

All statistical analyses were performed as appropriate to the experimental design using the SAS (Statistical Analysis Systems, Inc., Cary, NC) general linear models procedure (Schlotzauer and Littell 1987). Multiple comparisons between pairs of mean disease severity from different treatments using a series of t-tests followed SAS procedures in PROC ANOVA (Gomez and Gomez 1984; SAS/STAT 1990). The least significant differences (LSD) were calculated according to Fisher's protected LSD test at $P \leq 0.05$. Means followed by different letters demonstrated a statistical significant difference.

\section{RESULTS}

\section{Disease Development and Evaluation}

The first symptoms of bacterial blight caused by $P$. syringae pv. syringae were observed in April, soon after budbreak, and developed in severity until early to mid-May. Symptoms consisted of dark brown lesions on leaves and young stems. Actively growing plant parts were girdled, resulting in the death of terminal twigs. Infected leaves shriveled, as did most shoots and flower clusters (Figure 1a). New symptoms appeared throughout June, after which the infection rate began to decline. Blighted plants later recovered by producing new leaves, masking the initial damage. Throughout the study, bacterial blight symptoms were most prominent during the month of May. To confirm the causal agent of this disease, $P$. syringae pv. syringae was routinely isolated from blighted twigs and leaves. During this study, a total of 12 cultivars were found to be highly resistant and 15 to be susceptible to moderately susceptible (Table $1^{*}$ ).

Although the overall mean disease reading for $S$. vulgaris 'Avalanche', S. vulgaris 'Marie Legraye', and S. reticulata 'Ivory Silk' were low (0.0 to 2.0), they were categorized as moderately susceptible because they displayed high infection in one of the years of the study. During that year, they received scores higher than 2.5. Similarly, S. vulgaris 'Harry Bickle', S. vulgaris 'Silver King', S. hyacinthiflora 'Excel', and S. reticulata 'Summer Snow' were categorized as susceptible because they had disease readings of higher than 3 during one year (Table 1).

Symptoms of powdery mildew began to appear in July (1997, 1998, and 2002) and in June (1996). In response to humidity gradients, disease symptoms first appeared on the lower leaves then progressed to upper plant parts. Powdery mildew symptoms persisted throughout the growing season and increased in severity over time to reach their highest level in August (Figure 1b). A total of 29 cultivars (S. meyeri, S. prestoniae, S. josiflexa, S. hyacinthiflora, S. reticulata, S. microphylla, S. patula, S. henryi, and 13 cultivars of S. vulgaris) were resistant to powdery mildew; 19 cultivars of $S$. vulgaris and one of $S$. chinensis were susceptible or moderately susceptible (Table 2). Although some defoliation associated with severe powdery mildew infection (data not shown) was observed, the effect of this disease is mostly aesthetic and caused no significant difference in growth.

Beginning in mid-June to early July, severe leaf scorching

\footnotetext{
*Tables for this article appear on pp. 7-9.
} 


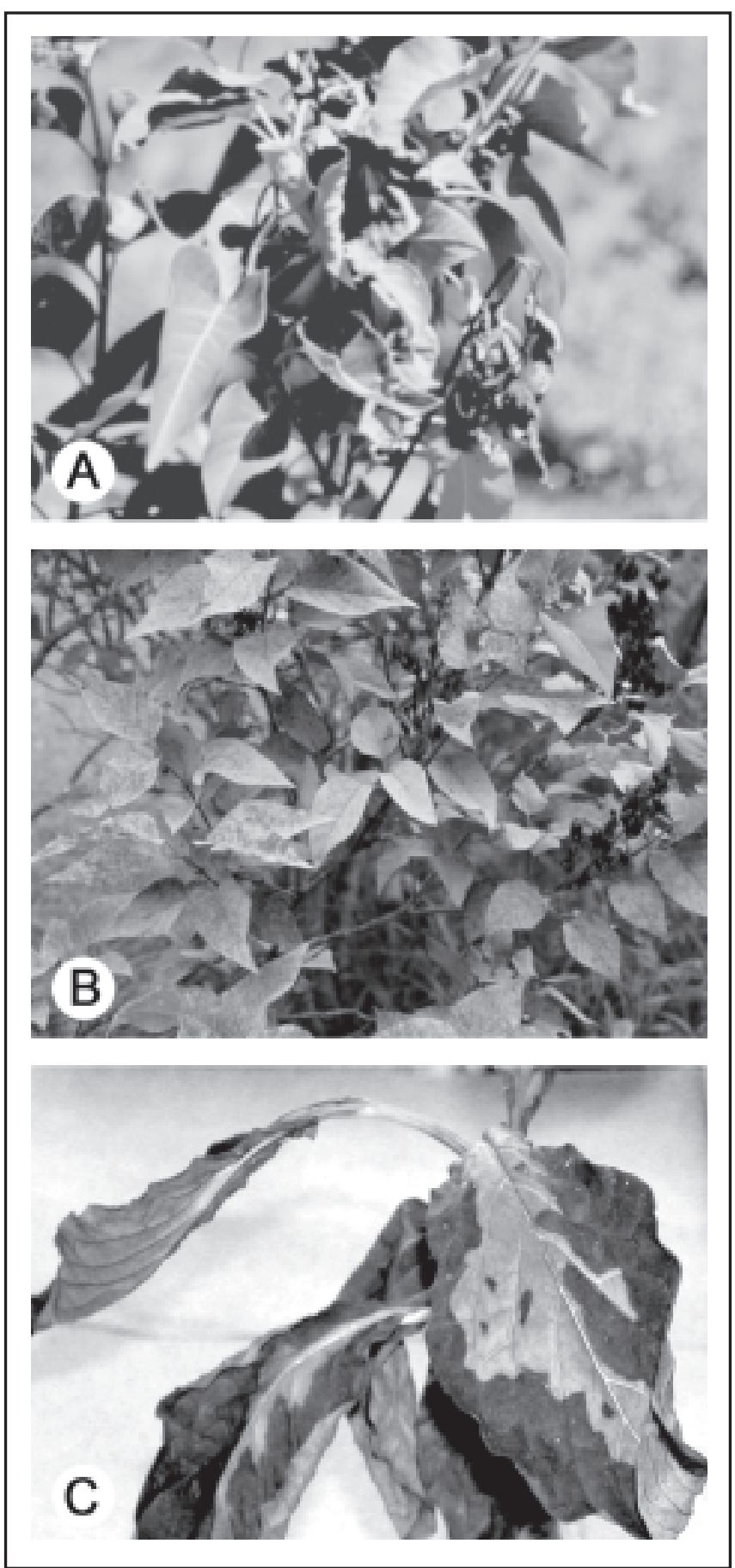

Figure 1. Lilac diseases evaluated during the study: (a) bacterial blight caused by Pseudomonas syringae pv. syringae, (b) powdery mildew caused by Microsphaera syringae, and (c) Alternaria blight caused by Alternaria alternata.

caused by A. alternata developed. Scorched areas consisted of brown, necrotic lesions with ash-colored centers (Figure 1c). In some taxa, the lesions were lighter in color, with or without stem lesions. Severely infected plants defoliated, losing 90\% to 95\% of their leaves by late July/early August. Some of the cultivars resistant to bacterial blight and/or to powdery mildew also defoliated. Only seven cultivars showed resistance or moderate resistance to A. alternata: $S$. meyeri 'Palibin' and 'Dwarf Korean', S. vulgaris 'Mme. Antoine Buchner' and 'Silver King', S. reticulata 'Ivory Silk' and ' Summer Snow', and S. hyacinthiflora 'Pocahontas' (Table 3).

Twenty-four cultivars were consistently resistant to bacterial blight and powdery mildew, six cultivars to powdery mildew and Alternaria blight, and four cultivars to bacterial blight and Alternaria blight (Table 4). Only two cultivars, S. meyeri 'Dwarf Korean' and S. meyeri 'Palibin', were resistant to all three diseases (Table 4).

\section{Alternaria}

Alternaria alternata was constantly isolated from diseased lilacs. The fungus was cultured on potato dextrose agar, and each isolate produced identical growth patterns and conidia. Disease-free plants of the same taxon were inoculated with spore suspensions prepared from a mixture of several isolates. The resulting disease symptoms on these plants were identical to those observed on the original plants from which the isolates were obtained. Re-isolation of the fungus from these plants yielded cultures identical to the originals. Conidia were dark in color, with the typical longitudinal and transverse septa along with the characteristic long beak found in the genus Alternaria (Barnett and Hunter 1998).

The following accessions were inoculated with spore suspensions prepared from the primary cultures: $S$. prestoniae 'Isabella', S. prestoniae 'James McFarlane', S. meyeri 'Dwarf Korean', and an unnamed S. vulgaris accession. All accessions developed identical symptoms as observed in the field. Symptom expression began to occur 6 days after inoculation and increased in severity for 10 weeks. Depending on the taxon, infected leaves developed brown, necrotic lesions with or without chlorotic bands and with or without concentric rings. Plants sprayed only with distilled water did not develop disease symptom.

\section{DISCUSSION}

Because field environment factors such as temperature and relative humidity play such an important role in disease development, the performance of a taxon over more than one growing season better reflected its ability to resist infection. For example, if a taxon had a disease rating of greater than 2.0 (moderately resistant) during the evaluation period, it was considered susceptible even if the 6-year mean average rating was less than 2.0 (resistant).

Powdery mildew-resistant cultivars such as such as $S$. prestoniae 'James McFarland' and S. patula 'Miss Kim' are available in Tennessee. These cultivars and others (Table 2) are good alternatives to mildew-susceptible common lilacs 
(S. vulgaris). Resistance to both powdery mildew and bacterial blight would allow for attractive foliage following flowering. Alternaria blight can have a devastating effect on the foliage (Figure 1c); if this disease occurs in conjunction with powdery mildew and/or bacterial blight, plants become much more unsightly by midsummer.

Powdery mildew and Alternaria blight were favored by the commonly warm and humid weather conditions of Tennessee. Throughout the study, Alternaria blight symptoms initially appeared on the upper or middle parts of the plants, sometimes on one side of the plant and subsequently spreading over the entire plant. Powdery mildew symptoms began on the lower leaves, progressing toward the upper parts. The mode of infection is typical of plant pathogens that persist between seasons on infected plants. Pschedt and Moorman (2001) reported that the bacterial blight pathogen in lilacs can overseason on infected twigs or as epiphytes on healthy-appearing wood.

It has not been demonstrated that powdery mildew can overseason, but it is believed to survive on infected leaf debris. Powdery mildew of lilacs forms abundant ascocarps beginning in July and germinates the following spring by liberating the ascospores. These ascospores apparently serve as the primary inoculum to initiate the disease cycle under favorable weather conditions. Variation in disease severity during a growing season is dependent on the prevailing temperature and relative humidity conditions and may change from season to season because temperature and humidity will affect the production and dispersal of secondary inoculum.

Many species of Alternaria are routinely found on decomposing plant material and on the leaf surfaces of most trees and shrubs (Sinclair et al. 1987). Most are nonpathogenic (Rotem 1994). The Alternaria isolate that we obtained from lilac was aggressive and highly pathogenic on susceptible lilacs (Mmbaga and Sheng 1987). Based on its morphology and cultural characteristics, this isolate was identified as A. alternata (Anderson and Thrane 1996). This species has a wide host range, causing leaf spots, blights, and blossom and fruit rot on many plant parts (Rotem 1994). More than 380 hosts have been recorded in the USDA Systematic Botany and Mycology Fungus-Host Distribution Database (http:// nt.ars-grin.gov). This genus is also considered to be one of the most important allergenic molds in the United States (Pharmacia Diagnostics 1992). Additional studies using RAPD-PCR analysis are ongoing to confirm our identification of this pathogen (Weir et al. 1998). We observed a wide difference in susceptibility among the lilac taxa in the study. In many cultivars, Alternaria blight was more damaging than bacterial blight or powdery mildew, and the combination as a disease complex with powdery mildew was synergistic. In those taxa, the pathological reaction to the two diseases as a complex was more severe than expected.
This study provides information on multiple disease resistance to the disease complex of powdery mildew, bacterial blight, and Alternaria blight and provides information on the performance of 56 commercial cultivars (Table 4). A few taxa had multiple disease resistance, while others were susceptible to all diseases (Table 4). Because multiple disease resistance occurs in this genus, new cultivars with multiple resistances could be developed by conventional breeding methods.

\section{LITERATURE CITED}

Anderson, B. and U. Thrane. 1996. Differentiation of Alternaria infectoria and Alternaria alternata based on morphology, metabolite profiles, and cultural characteristics. Can. J. Microbiol. 42:685-689.

Barnett, H.L., and B.B. Hunter. 1998. Illustrated Genera of Imperfect Fungi. APS Press, St. Paul, MN. 217 pp.

Clement, D.L., S.A. Gill, and W. Potts. 1994. Alternatives for powdery mildew control on lilacs. J. Arboric. 20:227-230.

Gomez, K.A., and A.A. Gomez. 1984. Statistical Procedures for Agricultural Research. John Wiley and Sons, New York, NY. 679 pp.

Hibben, C.R., J.T. Walker, and J.R. Allison 1977. Powdery mildew ratings of lilac species and cultivars. Plant Dis. Rep. 55:475-478.

Hildebrand, D.C., M.N. Schoroth, and D.C. Sands. 1988. Psudomonads, pp 60-80. In Schaad, N.W. (Ed.). Laboratory Guide for the Identification of Plant Pathogenic Bacteria. APS Press, St. Paul, MN. 158 pp.

Horsfall, J.G., and R.W. Barratt. 1945. An improved grading system for measuring plant disease. Phytopathology 35:655.

Jones, R.K. 1986. Powdery mildew, pp 24-25. In Jones, R.K. and R.C. Lambe. (Eds.). Diseases of Woody Ornamental Plants and Their Control in Nurseries. North Carolina Agricultural Extension Service, Raleigh, NC. 130 pp.

Koch, R. 1882. Über die Midzbrandimpfung: Eine Entgegnung auf den von Pasteur in Genf Gehaltenen Vortrag. Theodor Fischer, Kassel and Berlin, Germany.

Mmbaga, M.T., and H. Sheng. 1997. Evaluation of lilac (Syringa spp.) for multiple disease resistance to powdery mildew and bacterial blight in McMinnville, TN. Proc. South. Nurserymen's Assoc. Res. Conf. 42:512-518.

Mmbaga, M.T., E. Nnodu, and R.J. Sauvé. 2003. Alternaria blight in lilac. Proc. South. Nurserymen's Assoc. Res. Conf. 48:240-242.

Pharmacia Diagnostics. 1992. Alternaria alternata (A. tenius). www.unicapinvitrosight.com/templates/Allergens.asp?id= 2204.

Pschedt, J.W., and G.W. Moorman. 2001. Lilac diseases. In Jones, R.K., and D.M. Benson (Eds.). Diseases of Woody Ornamentals and Trees in Nurseries. APS Press, St. Paul, MN. 482 pp. 
Rotem, J. 1994. The Genus Alternaria: Biology, Epidemiology and Pathogenicity. APS Press, St. Paul, MN. 326 pp.

SAS/STAT. 1990. User's Guide, Vol. 1, Ver. 5 (4th ed.). SAS Institute, Cary, NC.

Schlotzauer, S.D., and R.C. Littell. 1987. SAS System for Elementary Statistical Analysis. SAS Institute, Cary, NC. $416 \mathrm{pp}$.

Sinclair, W.A., H. Lyon., and W.T. Johnson. 1987. Diseases of Trees and Shrubs. Cornell University Press, Ithaca, NY. 575 pp.

Smith, E.F. 1905. Bacteria in Relation to Plant Disease, Vol. 1. Carnegie Institution of Washington, Washington, DC.

Weir, T.L., D.R. Huff, B.J. Christ, and C.P. Romaine. 1998. RAPD-PCR analysis of genetic variation among isolates of Alternaria solani and A. alternata from potato and tomato. Mycologia 90:813821.

Widham, M.T., W.T. Witte. R.J. Sauvé, and P.C. Flanagan. 1995. Powdery mildew observations of lilac in Tennessee. Proc. Ann. Mtg. Am. Soc. Hortic. Sci. and Can. Soc. Hortic. Sci., Montréal, QC 92:454.

Acknowledgments. We thank Ms. Hogyan Sheng for her dedicated efforts in these studies. This work was partly funded by the USDA/CSREES Grant No. 98-38814 and by the Horticultural Research Institute.

\author{
${ }^{1,3}$ Nursery Crop Research Station \\ Tennessee State University \\ Institute of Agricultural and Environmental Research \\ McMinnville, TN 37110, U.S. \\ Immmbaga@tnstate.edu \\ 3ennodu@tnstate.edu \\ ${ }^{2,4}$ Tennessee State University \\ Institute of Agricultural and Environmental Research \\ 3500 John A. Merritt Blvd. \\ Nashville, TN 37209-1561, U.S. \\ 2rsauve@tnstate.edu \\ 33zsuping@tnstate.edu
}

"Corresponding author: Margaret T. Mmbaga.
Résumé. Cinquante-six cultivars de lilas ont été évalués au cours d'une étude de quatre ans en regard de la résistance au blanc des feuilles causée par Microsphaera syringae, à la brûlure bactérienne causée par Pseudomonas syringae pv. syringae et à la brûlure Alternaria causée par Alternaria alternata. Parmi les cultivars étudiés, 40 provenaient de Syringa vulgaris, quatre de $S$. prestioniae, trois de S. hyacingthiflora, deux de S. josiflexa, deux de S. meyeri, deux de S. reticulata, et un de S. patula, S. chinensis, S. henryi et $S$. mycrophylla. De ces derniers, six étaient résistants ou modérément résistants à la brûlure Alternaria et au blanc des feuilles, quatre à la brûlure bactérienne et la brûlure Alternaria, et 20 au blanc des feuilles et à la brûlure bactérienne. Deux cultivars de S. meyeri, 'Dwarf Korean' et 'Palibin', étaient résistants à ces trois maladies. Durant cette étude, la maladie la plus sérieuse sur les lilas du Tennessee central était la brûlure Alternaria. Elle a causé des brûlures au feuillage et des défoliations sévères qui ont débuté tôt en juin. Le blanc des feuilles et la brûlure bactérienne ont causé des dommages généralement superficiels avec peu d'incidence sur la croissance.

Zusammenfassung. 56 Flieder wurden in einer 4-jährigen Studie auf ihre Widerstandsfähigkeit gegenüber Mehltau, verursacht durch Microsphaera syringae, bakteriellen Brand, verursacht durch Pseudomonas syringae pv. Syringae und Alternaria Brand, verursacht durch Alternaria alternata untersucht. In der Auswahlgruppe waren 39 Kultivare von Syringa vulgaris, vier S. prestioniae, drei S. hyacinthiflora, zwei von S. josiflexa, zwei von S. meyeri, zwei von S. reticulata, und je ein S. patula, S. chinensis, $S$. henryi und S. microphylla. Von diesen waren sechs mehr oder weniger resistent gegenüber Alternaria-Brand und Mehltau, vier gegenüber bakteriellen Brand und Alternaria-Brand und 20 gegenüber Mehltau und bakteriellen Brand. Zwei Kultivare von S. meyeri ,Dwarf Korea' und ,Palibin' waren resistent gegenüber allen drei Pathogenen. Während dieser Studie war Alternaria-Brand für die Flieder in Mittel-Tennessee die gefährlichste Erkrankung. Er verursachte ernsthafte Blattflecken und eine im Juli einsetzende Entlaubung. Mehltau und bakterieller Brand verursachte überwiegend kosmetischen Schaden mit geringen Auswirkungen auf das Wachstum.

Resumen. Se evaluaron 56 especímenes de lila, en cuatro años de estudio, para estudiar la resistencia a la cenicilla, causada por Microsphaera syringae, al tizón bacterial, causado por Pseudomonas syringae pv. Syringae y a la alternaria, causada por Alternaria alternata. Los ejemplares incluyeron 39 cultivares de Syringa vulgaris, cuatro de S. prestioniae, tres de S. hyacingthiflora, dos de S. josiflexa, dos de S. meyeri, dos de S. reticulata, y uno de $S$. patula, S. chinensis, S. henryi, y de S. microphylla, respectivamente. De estos, seis ejemplares fueron resistentes o moderadamente resistentes a alternaria y cenicilla, cuatro al tizón bacterial y alternaria y 20 a la cenicilla y al tizón bacterial. Dos cultivares de $S$. meyeri, 'Dwarf Korean' y 'Palibin', fueron resistentes a los tres patógenos. Durante este estudio, la enfermedad más seria de la lila en Tennessee fue alternaria. Esta causó chamuscamiento severo y defoliación que empezó a inicios de Julio. La cenicilla y el tizón bacterial causaron principalmente daño superficial con poco efecto en el crecimiento. 
Table 1. Bacterial blight disease severity in lilacs over a 4-year period in middle Tennessee.

\begin{tabular}{|c|c|c|c|c|c|c|}
\hline \multirow[b]{2}{*}{ Syringa spp. } & \multirow[b]{2}{*}{ Cultivar } & \multicolumn{4}{|c|}{ Mean disease severity $(0-5 \text { scale })^{z}$} & \multirow{2}{*}{$\begin{array}{l}\text { Overall disease } \\
\text { reaction }^{y}\end{array}$} \\
\hline & & 1996 & 1997 & 1998 & 4-year mean & \\
\hline S. prestoniae & Donald Wyman & $0.1 \mathrm{n}$ & $0.00 \mathrm{~m}$ & $1.0 \mathrm{~g}-\mathrm{j}$ & 0.4 & $\mathrm{R}$ \\
\hline S. josiflexa & Royalty & $0.3 \mathrm{~m}-\mathrm{n}$ & $0.00 \mathrm{~m}$ & $0.8 \mathrm{i}-\mathrm{k}$ & 0.4 & $\mathrm{R}$ \\
\hline S. microphylla & Superba & - & $1.0 \mathrm{f}-\mathrm{k}$ & $0.0 \mathrm{k}$ & 0.5 & $\mathrm{R}$ \\
\hline S. patula & Miss Kim & $0.8 \mathrm{j}-\mathrm{n}$ & $1.0 \mathrm{f}-\mathrm{m}$ & $0.0 \mathrm{k}$ & 0.6 & $\mathrm{R}$ \\
\hline S. vulgaris & Edmund Boisier & $0.2 \mathrm{~m}-\mathrm{n}$ & 0.8 g-m & $0.9 \mathrm{~g}-\mathrm{k}$ & 0.6 & $\mathrm{R}$ \\
\hline S. vulgaris & Victor Lemoine & $0.31-n$ & $1.2 \mathrm{c}-1$ & - - & 0.8 & $\mathrm{R}$ \\
\hline S. meyeri & Dwarf Korean & 0.9 i-n & $1.2 \mathrm{~d}-1$ & $0.4 \mathrm{j}-\mathrm{k}$ & 0.8 & $\mathrm{R}$ \\
\hline S. vulgaris & Mme. Antoine Buchner & $1.5 \mathrm{f}-\mathrm{j}$ & 0.4 k-m & $0.8 \mathrm{i}-\mathrm{k}$ & 0.9 & $\mathrm{R}$ \\
\hline S. prestoniae & Isabella & 0.6 k-n & $1.0 \mathrm{f}-\mathrm{m}$ & $1.2 \mathrm{f}-\mathrm{j}$ & 0.9 & $\mathrm{R}$ \\
\hline S. vulgaris & Sensation & 1.0 i-m & 0.7 h-m & - & 0.9 & $\mathrm{R}$ \\
\hline S. josiflexa & Anna Amhof & $0.7 \mathrm{j}-\mathrm{n}$ & $0.5 \mathrm{j}-\mathrm{m}$ & $1.8 \mathrm{c}-\mathrm{h}$ & 1.0 & $\mathrm{R}$ \\
\hline S. vulgaris & Krasavitska Moskvy & $1.0 \mathrm{~h}-1$ & $0.6 \mathrm{i}-\mathrm{m}$ & $1.2 \mathrm{f}-\mathrm{j}$ & 1.0 & $\mathrm{R}$ \\
\hline S. vulgaris & Michael Buchner & $1.5 \mathrm{f}-\mathrm{j}$ & $0.2 \mathrm{~lm}$ & $1.2 \mathrm{f}-\mathrm{j}$ & 1.0 & $\mathrm{R}$ \\
\hline S. vulgaris & Alphonse Lavallée & 1.1 h-k & $1.0 \mathrm{f}-\mathrm{m}$ & 1.0 g-j & 1.0 & $\mathrm{R}$ \\
\hline S. vulgaris & Paul Thirion & $1.7 \mathrm{e}-\mathrm{i}$ & 0.7 h-m & $0.8 \mathrm{i}-\mathrm{k}$ & 1.1 & MR \\
\hline S. meyeri & Palibin & $1.8 \mathrm{~d}-\mathrm{h}$ & $1.0 \mathrm{f}-\mathrm{m}$ & 0.6 i-k & 1.1 & MR \\
\hline S. vulgaris & Charm & $1.0 \mathrm{i}-\mathrm{m}$ & 0.7 h-m & $1.5 \mathrm{~d}-\mathrm{i}$ & 1.1 & MR \\
\hline S. vulgaris & Marie Finon & $1.5 \mathrm{f}-\mathrm{j}$ & 0.7 h-m & —— & 1.1 & MR \\
\hline S. prestoniae & James McFarlane & $1.5 \mathrm{f}-\mathrm{j}$ & 0.6 i-m & $1.2 \mathrm{f}-\mathrm{j}$ & 1.1 & MR \\
\hline S. vulgaris & Yankee Doodle & 1.1 h-1 & $1.3 \mathrm{c}-\mathrm{k}$ & $1.2 \mathrm{f}-\mathrm{j}$ & 1.2 & MR \\
\hline S. vulgaris & Macrostachys & $0.8 \mathrm{j}-\mathrm{n}$ & 0.7 h-m & $2.0 \mathrm{~b}-\mathrm{f}$ & 1.2 & MR \\
\hline S. prestoniae & Minuet & $1.0 \mathrm{i}-\mathrm{m}$ & 0.4 k-m & $2.2 \mathrm{~b}-\mathrm{e}$ & 1.2 & MR \\
\hline S. hyacinthiflora & Sister Justena & $1.3 \mathrm{~g}-\mathrm{k}$ & $1.3 \mathrm{c}-\mathrm{k}$ & --- & 1.3 & MR \\
\hline S. vulgaris & Leon Gambetta & - - & $1.0 \mathrm{f}-\mathrm{m}$ & $1.5 \mathrm{~d}-\mathrm{i}$ & 1.3 & MR \\
\hline S. vulgaris & Mme. F Morel & $1.8 \mathrm{~d}-\mathrm{h}$ & 0.8 g-m & $1.2 \mathrm{f}-\mathrm{j}$ & 1.3 & MR \\
\hline S. vulgaris & Charles Tenth & $2.2 \mathrm{~b}-\mathrm{f}$ & $1.0 \mathrm{f}-\mathrm{g}-\mathrm{m}$ & $0.8 \mathrm{i}-\mathrm{k}$ & 1.3 & MR \\
\hline S. vulgaris & Adelaide Dunbar & $1.0 \mathrm{i}-\mathrm{m}$ & $1.5 \mathrm{c}-\mathrm{ij}$ & $1.8 \mathrm{c}-\mathrm{h}$ & 1.4 & MR \\
\hline S. vulgaris & Henri Robert & 1.0 i-m & $1.9 \mathrm{c}-\mathrm{g}$ & - - & 1.4 & MR \\
\hline S. vulgaris & President Poincare & 1.3 g-k & $1.1 \mathrm{e}-1$ & $1.8 \mathrm{c}-\mathrm{g}$ & 1.4 & MR \\
\hline S. vulgaris & Charles Joly & $1.1 \mathrm{~h}-1$ & $1.7 \mathrm{c}-\mathrm{h}$ & - $^{\circ}$ & 1.4 & MR \\
\hline S. chinensis & Rothomagensis & $1.5 \mathrm{f}-\mathrm{ij}$ & $1.7 \mathrm{c}-\mathrm{h}$ & $1.3 \mathrm{f}-\mathrm{j}$ & 1.5 & MR \\
\hline S. vulgaris & Ludwig Spaeth & $2.2 \mathrm{~b}-\mathrm{f}$ & 0.7 h-m & $-\ldots$ & 1.5 & MR \\
\hline S. vulgaris & Belle de Nancy & $2.2 \mathrm{~b}-\mathrm{f}$ & $1.0 \mathrm{f}-\mathrm{m}$ & $1.4 \mathrm{e}-\mathrm{i}$ & 1.5 & MR \\
\hline S. vulgaris & Montaigne & $1.7 \mathrm{e}-\mathrm{i}$ & $1.0 \mathrm{f}-\mathrm{m}$ & $2.0 \mathrm{~b}-\mathrm{f}$ & 1.6 & MR \\
\hline S. vulgaris & Albert F. Holden & 0.6 k-n & $2.0 \mathrm{c}-\mathrm{f}$ & $2.2 \mathrm{~b}-\mathrm{e}$ & 1.6 & MR \\
\hline S. henryi & White Summers & $1.5 \mathrm{f}-\mathrm{j}$ & $1.6 \mathrm{c}-\mathrm{i}$ & $2.0 \mathrm{~b}-\mathrm{f}$ & 1.7 & MR \\
\hline S. vulgaris & Miss Ellen Wilmott & $1.5 \mathrm{f}-\mathrm{j}$ & $1.5 \mathrm{c}-\mathrm{j}$ & $2.2 \mathrm{~b}-\mathrm{e}$ & 1.7 & MR \\
\hline S. vulgaris & President Lincoln & $1.5 \mathrm{f}-\mathrm{j}$ & $1.8 \mathrm{c}-\mathrm{g}$ & $2.1 \mathrm{~b}-\mathrm{f}$ & 1.8 & MR \\
\hline S. vulgaris & Vestale & $2.2 \mathrm{~b}-\mathrm{f}$ & $1.0 \mathrm{f}-\mathrm{m}$ & $2.0 \mathrm{~b}-\mathrm{f}$ & 1.7 & MS \\
\hline S. vulgaris & Edith Cavell & $2.2 \mathrm{~b}-\mathrm{f}$ & $1.5 \mathrm{c}-\mathrm{j}$ & $1.5 \mathrm{~d}-\mathrm{i}$ & 1.7 & MR \\
\hline S. vulgaris & Avalanche & $2.5 \mathrm{a}-\mathrm{d}$ & $1.0 \mathrm{f}-\mathrm{m}$ & $1.8 \mathrm{c}-\mathrm{h}$ & 1.8 & MS \\
\hline S. vulgaris & Firmament & $2.0 \mathrm{c}-\mathrm{g}$ & $2.2 \mathrm{~b}-\mathrm{d}$ & $2.0 \mathrm{~b}-\mathrm{f}$ & 2.0 & MS \\
\hline S. reticulata & Ivory Silk & $2.7 \mathrm{a}-\mathrm{c}$ & $1.3 \mathrm{c}-\mathrm{k}$ & $2.0 \mathrm{~b}-\mathrm{f}$ & 2.0 & MS \\
\hline S. vulgaris & Marie Legraye & $2.2 \mathrm{~b}-\mathrm{f}$ & $1.1 \mathrm{e}-1$ & $2.8 \mathrm{a}-\mathrm{b}$ & 2.0 & MS \\
\hline S. hyacinthiflora & Pocahontas & 2.2 b-f & $2.0 \mathrm{c}-\mathrm{f}$ & $2.2 \mathrm{~b}-\mathrm{e}$ & 2.1 & MS \\
\hline S. vulgaris & Monge & $2.5 \mathrm{a}-\mathrm{d}$ & $2.0 \mathrm{c}-\mathrm{f}$ & $1.8 \mathrm{c}-\mathrm{h}$ & 2.1 & MS \\
\hline S. vulgaris & President Grevy & $2.5 \mathrm{a}-\mathrm{d}$ & $1.9 \mathrm{c}-\mathrm{g}$ & $1.8 \mathrm{c}-\mathrm{h}$ & 2.1 & MS \\
\hline S. vulgaris & A.M. Brand & $2.2 \mathrm{~b}-\mathrm{f}$ & $2.1 \mathrm{~b}-\mathrm{e}$ & $2.4 \mathrm{a}-\mathrm{d}$ & 2.2 & MS \\
\hline S. vulgaris & Ruhm Von Horstenstein & $2.7 \mathrm{a}-\mathrm{c}$ & $1.7 \mathrm{c}-\mathrm{h}$ & $2.3 \mathrm{~b}-\mathrm{e}$ & 2.2 & MS \\
\hline S. vulgaris & Arch McKean & $2.3 \mathrm{~b}-\mathrm{e}$ & $2.2 \mathrm{~b}-\mathrm{d}$ & $2.5 \mathrm{a}-\mathrm{c}$ & 2.4 & MS \\
\hline S. vulgaris & Mrs. W.E. Marshall & $2.7 \mathrm{a}-\mathrm{c}$ & $2.2 \mathrm{~b}-\mathrm{d}$ & - - & 2.4 & MS \\
\hline S. vulgaris & Silver King & 1.9 d-h & $3.2 \mathrm{a}$ & $2.4 \mathrm{a}-\mathrm{d}$ & 2.5 & S \\
\hline S. hyacinthiflora & Excel & $1.5 \mathrm{f}-\mathrm{j}$ & $3.1 \mathrm{a}-\mathrm{b}$ & $2.8 \mathrm{a}-\mathrm{b}$ & 2.5 & S \\
\hline S. vulgaris & Mrs. Harry Bickle & $3.2 \mathrm{a}$ & $2.2 \mathrm{~b}-\mathrm{d}$ & - & 2.7 & $\mathrm{~S}$ \\
\hline S. reticulata & Summer Snow & $3.2 \mathrm{a}$ & $2.3 \mathrm{~b}-\mathrm{c}$ & - & 2.7 & $\mathrm{~S}$ \\
\hline S. vulgaris & Katherine Havemeyer & $2.8 \mathrm{a}-\mathrm{b}$ & $2.2 \mathrm{~b}-\mathrm{d}$ & $3.4 \mathrm{a}$ & 2.8 & S \\
\hline $\mathrm{LSD}_{(0.05)}$ & & 0.8 & 1.1 & 1.1 & & \\
\hline
\end{tabular}

${ }^{2}$ Disease readings of $0=$ no symptoms, $1=10 \%, 2=11 \%$ to $25 \%, 3=26 \%$ to $50 \%, 4=51 \%$ to $75 \%$, and $5=76 \%$ to $100 \%$ of the foliage showing disease symptoms. y Disease reactions categorized as resistant (R), moderately resistant (MR), moderately susceptible (MS), or susceptible (S) were based on the mean disease readings obtained during the growing season in which $\mathrm{R}=0$ to $1.0, \mathrm{MR}=1.1$ to $2.0, \mathrm{MS}=2.1$ to 2.9 , and $\mathrm{S}=3.0$ to 5.0 . Disease readings greater than 2.0 during any growing season disqualified a cultivar from being considered resistant even if the 4-year mean disease reading was less than 2.0; any disease reading of 2.5 to 3.0 during any growing season was an indication of moderate susceptibility, and any reading of greater than 3.0 during a growing season was regarded as fully susceptible. 
Table 2. Powdery mildew disease severity in lilacs over a 4-year period in middle Tennessee.

\begin{tabular}{|c|c|c|c|c|c|c|c|}
\hline Syringa spp. & Cultivar & \multicolumn{4}{|c|}{ Mean disease severity $(0-5 \text { scale })^{z}$} & 4-year mean & $\begin{array}{l}\text { Overall disease } \\
\text { reaction }\end{array}$ \\
\hline S. hyacinthiflora & Sister Justena & $0.0 \mathrm{j}$ & $0.0 \mathrm{~h}$ & - - & - - & 0 & $\mathrm{R}$ \\
\hline S. josiflexa & Royalty & $0.0 \mathrm{j}$ & $0.0 \mathrm{~h}$ & 0.01 & $0.0 \mathrm{k}$ & 0 & $\mathrm{R}$ \\
\hline S. josiflexa & Anna Amhof & $0.0 \mathrm{j}$ & $0.0 \mathrm{~h}$ & 0.01 & $0.0 \mathrm{k}$ & 0 & $\mathrm{R}$ \\
\hline S. vulgaris & Marie Finon & $0.0 \mathrm{j}$ & $0.0 \mathrm{~h}$ & - - & - - & 0 & $\mathrm{R}$ \\
\hline S. prestoniae & Minuet & $0.0 \mathrm{j}$ & $0.0 \mathrm{~h}$ & 0.01 & - & 0 & $\mathrm{R}$ \\
\hline S. prestoniae & James McFarlane & $0.0 \mathrm{j}$ & $0.0 \mathrm{~h}$ & 0.01 & - & 0 & $\mathrm{R}$ \\
\hline S. prestoniae & Donald Wyman & $0.0 \mathrm{j}$ & $0.0 \mathrm{~h}$ & 0.01 & - & 0 & $\mathrm{R}$ \\
\hline S. prestoniae & Isabella & $0.0 \mathrm{j}$ & $0.0 \mathrm{~h}$ & 0.01 & $0.0 \mathrm{k}$ & 0 & $\mathrm{R}$ \\
\hline S. reticulata & Summer Snow & $0.0 \mathrm{j}$ & $0.0 \mathrm{~h}$ & - & - & 0 & $\mathrm{R}$ \\
\hline S. vulgaris & Sensation & $0.0 \mathrm{j}$ & $0.0 \mathrm{~h}$ & - & - & 0 & $\mathrm{R}$ \\
\hline S. henryi & White Summers & $0.0 \mathrm{j}$ & $0.0 \mathrm{~h}$ & $0.2 \mathrm{k}-1$ & $0.0 \mathrm{k}$ & 0.1 & $\mathrm{R}$ \\
\hline S. meyeri & Palibin & $0.0 \mathrm{j}$ & $0.0 \mathrm{~h}$ & 0.01 & $0.3 \mathrm{j}-\mathrm{k}$ & 0.1 & $\mathrm{R}$ \\
\hline S. meyeri & Dwarf Korean & $0.0 \mathrm{j}$ & $0.0 \mathrm{~h}$ & 0.01 & $0.7 \mathrm{i}-\mathrm{k}$ & 0.1 & $\mathrm{R}$ \\
\hline S. reticulata & Ivory Silk & $0.0 \mathrm{j}$ & $0.0 \mathrm{~h}$ & 0.01 & $0.3 \mathrm{j}-\mathrm{k}$ & 0.1 & $\mathrm{R}$ \\
\hline S. vulgaris & Firmament & $0.0 \mathrm{j}$ & $0.4 \mathrm{~g}-\mathrm{h}$ & 0.01 & $0.3 \mathrm{j}-\mathrm{k}$ & 0.2 & $\mathrm{R}$ \\
\hline S. microphylla & Superba & - & $0.0 \mathrm{~h}$ & 0.4 k-1 & $0.3 \mathrm{j}-\mathrm{k}$ & 0.2 & $\mathrm{R}$ \\
\hline S. patula & Miss Kim & $0.0 \mathrm{j}$ & $0.0 \mathrm{~h}$ & 0.01 & $1.3 \mathrm{~g}-\mathrm{k}$ & 0.3 & $\mathrm{R}$ \\
\hline S. vulgaris & Charm & $0.0 \mathrm{j}$ & $0.0 \mathrm{~h}$ & $0.8 \mathrm{i}-1$ & - - & 0.3 & $\mathrm{R}$ \\
\hline S. vulgaris & Edith Cavell & $0.0 \mathrm{j}$ & $0.0 \mathrm{~h}$ & 1.2 h-1 & $0.0 \mathrm{k}$ & 0.3 & $\mathrm{R}$ \\
\hline S. vulgaris & Mrs. Harry Bickle & $1.3 \mathrm{f}-\mathrm{j}$ & $0.0 \mathrm{~h}$ & - - & $0.3 \mathrm{jk}$ & 0.5 & $\mathrm{R}$ \\
\hline S. vulgaris & Henri Robert & $0.0 \mathrm{j}$ & $1.0 \mathrm{~d}-\mathrm{h}$ & - - & - & 0.5 & $\mathrm{R}$ \\
\hline S. hyacinthiflora & Excel & $0.7 \mathrm{~h}-\mathrm{j}$ & $0.0 \mathrm{~h}$ & $0.4 \mathrm{kl}$ & $1.3 \mathrm{~g}-\mathrm{k}$ & 0.6 & $\mathrm{R}$ \\
\hline S. vulgaris & Marie Legraye & $0.0 \mathrm{j}$ & $0.0 \mathrm{~h}$ & $0.6 \mathrm{j}-1$ & $2.0 \mathrm{e}-\mathrm{i}$ & 0.6 & $\mathrm{R}$ \\
\hline S. vulgaris & Vestale & $1.7 \mathrm{e}-\mathrm{i}$ & $0.4 \mathrm{~g}-\mathrm{h}$ & $0.8 \mathrm{i}-1$ & $0.5 \mathrm{i}-\mathrm{k}$ & 0.7 & $\mathrm{R}$ \\
\hline S. vulgaris & Arch McKean & $0.0 \mathrm{j}$ & $0.0 \mathrm{~h}$ & 0.01 & $0.3 \mathrm{j}-\mathrm{k}$ & 0.9 & $\mathrm{R}$ \\
\hline S. hyacinthiflora & Pocahontas & $0.7 \mathrm{~h}-\mathrm{j}$ & $0.8 \mathrm{e}-\mathrm{h}$ & $0.6 \mathrm{j}-1$ & $2.0 \mathrm{e}-\mathrm{i}$ & 1.0 & $\mathrm{R}$ \\
\hline S. vulgaris & Ludwig Spaeth & $1.3 \mathrm{f}-\mathrm{j}$ & $0.7 \mathrm{e}-\mathrm{h}$ & -- & - - & 1.0 & $\mathrm{R}$ \\
\hline S. vulgaris & Macrostachys & $1.0 \mathrm{~g}-\mathrm{j}$ & $0.0 \mathrm{~h}$ & $1.6 \mathrm{f}-\mathrm{k}$ & $2.0 \mathrm{e}-\mathrm{i}$ & 1.2 & $\mathrm{R}$ \\
\hline S. vulgaris & President Lincoln & $0.7 \mathrm{~h}-\mathrm{j}$ & $0.6 \mathrm{f}-\mathrm{h}$ & $2.0 e-j$ & $2.0 \mathrm{e}-\mathrm{i}$ & 1.3 & $\mathrm{R}$ \\
\hline S. vulgaris & Katherine Havemeyer & $0.0 \mathrm{j}$ & $0.0 \mathrm{~h}$ & $2.2 \mathrm{~d}-\mathrm{i}$ & $1.0 \mathrm{~h}-\mathrm{k}$ & 0.8 & MR \\
\hline S. vulgaris & Avalanche & $0.0 \mathrm{j}$ & $0.0 \mathrm{~h}$ & 1.2 h-1 & $2.3 \mathrm{~d}-\mathrm{h}$ & 0.9 & MR \\
\hline S. vulgaris & Charles Joly & $0.7 \mathrm{~h}-\mathrm{j}$ & $0.0 \mathrm{~h}$ & $2.2 \mathrm{~d}-\mathrm{i}$ & $2.0 \mathrm{e}-\mathrm{i}$ & 1.2 & MR \\
\hline S. vulgaris & Paul Thirion & $2.3 \mathrm{~d}-\mathrm{g}$ & $0.5 \mathrm{f}-\mathrm{h}$ & 1.2 h-1 & $1.7 \mathrm{f}-\mathrm{j}$ & 1.4 & MR \\
\hline S. vulgaris & Silver King & $1.3 \mathrm{f}-\mathrm{j}$ & $0.6 \mathrm{f}-\mathrm{h}$ & 1.0 i-1 & $2.3 \mathrm{~d}-\mathrm{h}$ & 1.4 & MR \\
\hline S. vulgaris & Mme. F Morel & $1.3 \mathrm{f}-\mathrm{j}$ & $0.2 \mathrm{~g}-\mathrm{h}$ & $2.2 \mathrm{~d}-\mathrm{i}$ & $1.7 \mathrm{f}-\mathrm{j}$ & 1.4 & MR \\
\hline S. vulgaris & President Poincare & $0.0 \mathrm{j}$ & $0.4 \mathrm{~g}-\mathrm{h}$ & $1.4 \mathrm{~g}-1$ & $2.7 \mathrm{c}-\mathrm{g}$ & 1.1 & MS \\
\hline S. vulgaris & Albert F. Holden & $0.3 \mathrm{i}-\mathrm{j}$ & $0.4 \mathrm{~g}-\mathrm{h}$ & $1.4 \mathrm{~g}-1$ & $2.7 \mathrm{c}-\mathrm{g}$ & 1.1 & MS \\
\hline S. vulgaris & Alphonse Lavallée & $0.3 \mathrm{i}-\mathrm{j}$ & $1.0 \mathrm{~d}-\mathrm{h}$ & $2.8 \mathrm{c}-\mathrm{g}$ & $1.3 \mathrm{~g}-\mathrm{k}$ & 1.4 & MS \\
\hline S. vulgaris & Miss Ellen Wilmott & $1.3 \mathrm{f}-\mathrm{j}$ & $0.0 \mathrm{~h}$ & $3.0 \mathrm{~b}-\mathrm{f}$ & $2.3 \mathrm{~d}-\mathrm{h}$ & 1.7 & MS \\
\hline S. vulgaris & Michael Buchner & $2.7 \mathrm{c}-\mathrm{f}$ & $0.2 \mathrm{~g}-\mathrm{h}$ & $2.8 \mathrm{c}-\mathrm{g}$ & $2.7 \mathrm{c}-\mathrm{g}$ & 2.0 & MS \\
\hline S. vulgaris & A.M. Brand & $2.7 \mathrm{c}-\mathrm{f}$ & $1.8 \mathrm{c}-\mathrm{e}$ & $1.2 \mathrm{~h}-1$ & $2.7 \mathrm{c}-\mathrm{g}$ & 2.0 & MS \\
\hline S. vulgaris & Leon Gambetta & - & $1.2 \mathrm{c}-\mathrm{g}$ & - & $3.0 \mathrm{~b}-\mathrm{f}$ & 2.1 & MS \\
\hline S. vulgaris & Victor Lemoine & $2.7 \mathrm{c}-\mathrm{f}$ & $2.0 \mathrm{c}-\mathrm{d}$ & - - & - - & 2.2 & MS \\
\hline S. vulgaris & Krasavitska Moskvy & $1.7 \mathrm{e}-\mathrm{i}$ & $1.2 \mathrm{~d}-\mathrm{g}$ & $1.6 \mathrm{f}-\mathrm{k}$ & 3.3 a-e & 1.9 & $\mathrm{~S}$ \\
\hline S. vulgaris & Ruhm Von Horstenstein & $1.0 \mathrm{~g}-\mathrm{j}$ & $1.0 \mathrm{~d}-\mathrm{h}$ & $2.2 \mathrm{~d}-\mathrm{i}$ & 3.3 a-e & 1.9 & S \\
\hline S. vulgaris & Montaigne & $0.3 \mathrm{i}-\mathrm{j}$ & $1.2 \mathrm{~d}-\mathrm{g}$ & $2.6 \mathrm{~d}-\mathrm{h}$ & 3.7 a-d & 2.0 & S \\
\hline S. vulgaris & Edmund Boisier & $2.0 \mathrm{~d}-\mathrm{h}$ & $1.2 \mathrm{~d}-\mathrm{g}$ & $3.6 \mathrm{a}-\mathrm{d}$ & $4.0 \mathrm{a}-\mathrm{c}$ & 2.5 & S \\
\hline S. vulgaris & Adelaide Dunbar & $1.7 \mathrm{e}-\mathrm{i}$ & $1.0 \mathrm{~d}-\mathrm{h}$ & $3.2 \mathrm{~b}-\mathrm{e}$ & $4.3 a-b$ & 2.6 & S \\
\hline S. vulgaris & Yankee Doodle & $0.3 \mathrm{i}-\mathrm{j}$ & $0.2 \mathrm{~g}-\mathrm{h}$ & $2.2 \mathrm{~d}-\mathrm{i}$ & 3.7 a-d & 2.6 & S \\
\hline S. vulgaris & Belle de Nancy & $1.7 \mathrm{e}-\mathrm{i}$ & $1.8 \mathrm{c}-\mathrm{e}$ & 3.4 a-e & $4.0 \mathrm{a}-\mathrm{c}$ & 2.7 & S \\
\hline S. vulgaris & Charles Tenth & $3.3 \mathrm{~b}-\mathrm{d}$ & $2.0 \mathrm{c}-\mathrm{d}$ & $2.6 \mathrm{~d}-\mathrm{h}$ & $4.3 a-b$ & 3.1 & S \\
\hline S. vulgaris & Mrs. W.E. Marshall & $4.0 \mathrm{a}-\mathrm{c}$ & $2.0 \mathrm{c}-\mathrm{d}$ & - & $4.3 a-b$ & 3.5 & S \\
\hline S. vulgaris & Monge & $3.0 \mathrm{~b}-\mathrm{e}$ & $3.2 a-b$ & $3.6 \mathrm{a}-\mathrm{d}$ & $4.7 \mathrm{a}$ & 3.6 & S \\
\hline S. chinensis & Rothomagensis & $3.3 \mathrm{~b}-\mathrm{d}$ & $3.5 a-b$ & $4.2 \mathrm{a}-\mathrm{c}$ & $4.0 \mathrm{a}-\mathrm{c}$ & 3.8 & S \\
\hline S. vulgaris & Mme. Antoine Buchner & $5.0 \mathrm{a}$ & $2.4 \mathrm{~b}-\mathrm{c}$ & $4.4 a-b$ & $2.7 \mathrm{c}-\mathrm{g}$ & 4.1 & S \\
\hline S. vulgaris & President Grevy & $4.3 a-b$ & $4.0 \mathrm{a}$ & $4.8 \mathrm{a}$ & $4.7 \mathrm{a}^{\circ}$ & 4.5 & S \\
\hline $\operatorname{LSD}_{(0.05)}$ & & 1.2 & 1.1 & 1.5 & 1.5 & & \\
\hline
\end{tabular}

${ }^{2}$ Disease readings of $0=$ no symptoms, $1=10 \%, 2=11 \%$ to $25 \%, 3=26 \%$ to $50 \%, 4=51 \%$ to $75 \%$, and $5=76 \%$ to $100 \%$ of the foliage showing disease symptoms

'Disease reactions categorized as resistant (R), moderately resistant (MR), moderately susceptible (MS), or susceptible (S) were based on the mean disease readings obtained during the growing season in which $\mathrm{R}=0$ to $1.0, \mathrm{MR}=1.1$ to $2.0, \mathrm{MS}=2.1$ to 2.9 , and $\mathrm{S}=3.0$ to 5.0 . Disease readings greater than 2.0 during any growing season disqualified a cultivar from being considered resistant even if the 4-year mean disease reading was less than 2.0 ; any disease reading of 2.5 to 3.0 during any growing season was an indication of moderate susceptibility, and any reading of greater than 3.0 during a growing season was regarded as fully susceptible. 
Table 3. Alternaria blight disease severity in lilacs over a 4-year period in middle Tennessee.

\begin{tabular}{|c|c|c|c|c|c|c|c|}
\hline \multirow[b]{2}{*}{ Syringa spp. } & \multirow[b]{2}{*}{ Cultivar } & \multicolumn{5}{|c|}{ Mean disease severity $(0-5 \text { scale })^{z}$} & \multirow[b]{2}{*}{$\begin{array}{l}\text { Overall disease } \\
\text { reaction }\end{array}$} \\
\hline & & 1996 & 1997 & 1998 & 2002 & $\begin{array}{l}\text { 2- to 4-year } \\
\text { mean }\end{array}$ & \\
\hline S. meyeri & Palibin & $0.3 \mathrm{k}$ & $1.2 \mathrm{i}-\mathrm{k}$ & $0.0 \mathrm{~m}$ & $1.0 \mathrm{j}-\mathrm{k}$ & 0.6 & $\mathrm{R}$ \\
\hline S. meyeri & Dwarf Korean & $0.7 \mathrm{j}-\mathrm{k}$ & $0.8 \mathrm{j}-\mathrm{k}$ & $0.0 \mathrm{~m}$ & $1.7 \mathrm{~h}-\mathrm{k}$ & 0.8 & $\mathrm{R}$ \\
\hline S. vulgaris & Mme. Antoine Buchner & $1.0 \mathrm{i}-\mathrm{k}$ & $1.2 \mathrm{i}-\mathrm{k}$ & $0.6 \mathrm{j}-\mathrm{m}$ & 1.3 i-k & 1.0 & $\mathrm{R}$ \\
\hline S. vulgaris & Silver King & $1.7 \mathrm{~h}-\mathrm{j}$ & $1.2 \mathrm{i}-\mathrm{k}$ & $1.0 \mathrm{~h}-\mathrm{m}$ & $1.0 \mathrm{j}-\mathrm{k}$ & 1.2 & MR \\
\hline S. reticulata & Ivory Silk & $2.0 \mathrm{~g}-\mathrm{i}$ & $1.6 \mathrm{f}-\mathrm{j}$ & $0.6 \mathrm{j}-\mathrm{m}$ & $1.0 \mathrm{j}-\mathrm{k}$ & 1.3 & MR \\
\hline S. reticulata & Summer Snow & $1.7 \mathrm{~h}-\mathrm{j}$ & $1.7 \mathrm{e}-\mathrm{j}$ & - & - & 1.7 & MR \\
\hline S. hyacinthiflora & Pocahontas & $2.0 \mathrm{~g}-\mathrm{i}$ & $1.8 \mathrm{e}-\mathrm{j}$ & $1.8 \mathrm{~d}-\mathrm{j}$ & $2.0 \mathrm{~g}-\mathrm{j}$ & 1.9 & MR \\
\hline S. vulgaris & Leon Gambetta & - & $1.7 e-j$ & - - & $2.3 \mathrm{f}-\mathrm{i}$ & 2.0 & MR \\
\hline S. vulgaris & Henry Robert & $2.3 \mathrm{c}-\mathrm{f}$ & $1.5 \mathrm{f}-\mathrm{j}$ & - - & - - & 1.9 & MR \\
\hline S. vulgaris & Sensation & $2.5 \mathrm{~b}-\mathrm{f}$ & $1.2 \mathrm{~h}-\mathrm{k}$ & - & - & 1.9 & MR \\
\hline S. microphylla & Superba & - & $0.0 \mathrm{k}$ & $2.0 \mathrm{c}-\mathrm{i}$ & $2.7 \mathrm{e}-\mathrm{h}$ & 1.6 & MS \\
\hline S. vulgaris & Michael Buchner & $2.7 \mathrm{e}-\mathrm{h}$ & $1.8 \mathrm{j}-\mathrm{k}$ & $1.0 \mathrm{~h}-\mathrm{m}$ & $2.3 \mathrm{f}-\mathrm{i}$ & 1.9 & MS \\
\hline S. vulgaris & Firmament & $2.7 \mathrm{e}-\mathrm{h}$ & $2.0 \mathrm{~d}-\mathrm{j}$ & $0.21-\mathrm{m}$ & $2.7 \mathrm{e}-\mathrm{h}$ & 1.9 & MS \\
\hline S. vulgaris & Paul Thirion & $2.7 \mathrm{e}-\mathrm{h}$ & $1.7 \mathrm{e}-\mathrm{j}$ & $1.2 \mathrm{~g}-\mathrm{m}$ & $2.0 \mathrm{~g}-\mathrm{j}$ & 1.9 & MS \\
\hline S. vulgaris & Charm & $2.7 \mathrm{e}-\mathrm{h}$ & $2.7 \mathrm{~b}-\mathrm{g}$ & $1.2 \mathrm{~g}-\mathrm{m}$ & - - & 2.0 & MS \\
\hline S. hyacinthiflora & Excel & $2.7 \mathrm{e}-\mathrm{h}$ & $1.8 \mathrm{e}-\mathrm{j}$ & $0.4 \mathrm{k}-\mathrm{m}$ & $3.0 \mathrm{~d}-\mathrm{g}$ & 2.0 & MS \\
\hline S. vulgaris & Yankee Doodle & $2.7 \mathrm{a}-\mathrm{b}$ & $1.8 \mathrm{e}-\mathrm{j}$ & $1.2 \mathrm{~g}-\mathrm{m}$ & $2.7 \mathrm{e}-\mathrm{h}$ & 2.1 & MS \\
\hline S. josiflexa & Royalty & $2.9 \mathrm{~d}-\mathrm{g}$ & $2.6 \mathrm{c}-\mathrm{h}$ & $1.2 \mathrm{~g}-\mathrm{m}$ & $2.0 \mathrm{~g}-\mathrm{j}$ & 2.2 & MS \\
\hline S. vulgaris & Ludwig Spaeth & $2.7 \mathrm{a}-\mathrm{b}$ & $2.0 \mathrm{~d}-\mathrm{j}$ & - & - & 2.3 & $\mathrm{~S}$ \\
\hline S. hyacinthiflora & Sister Justena & $2.9 \mathrm{~d}-\mathrm{g}$ & $1.7 \mathrm{e}-\mathrm{j}$ & - - & - & 2.3 & S \\
\hline S. vulgaris & Ruhm Von Horstenstein & $2.3 \mathrm{f}-\mathrm{h}$ & $1.2 \mathrm{~h}-\mathrm{k}$ & 2.8 a-e & $2.7 \mathrm{e}-\mathrm{h}$ & 2.3 & S \\
\hline S. vulgaris & Macrostachys & $3.0 \mathrm{~d}-\mathrm{g}$ & $2.7 \mathrm{~b}-\mathrm{g}$ & $2.2 \mathrm{~b}-\mathrm{h}$ & $1.7 \mathrm{~h}-\mathrm{k}$ & 2.4 & S \\
\hline S. vulgaris & Belle de Nancy & $3.7 \mathrm{~b}-\mathrm{e}$ & $2.2 \mathrm{c}-\mathrm{i}$ & $0.8 \mathrm{i}-\mathrm{m}$ & $2.7 \mathrm{e}-\mathrm{h}$ & 2.3 & S \\
\hline S. vulgaris & Albert F. Holden & $4.3 \mathrm{a}-\mathrm{c}$ & $1.8 e-j$ & $0.8 \mathrm{i}-\mathrm{m}$ & $3.0 \mathrm{~d}-\mathrm{g}$ & 2.3 & S \\
\hline S. prestoniae & Donald Wyman & $3.3 \mathrm{c}-\mathrm{f}$ & $2.2 \mathrm{c}-\mathrm{i}$ & $1.4 \mathrm{f}-1$ & - - & 2.3 & S \\
\hline S. vulgaris & Miss Ellen Wilmott & $3.0 \mathrm{~d}-\mathrm{g}$ & $1.8 \mathrm{e}-\mathrm{j}$ & $1.2 \mathrm{~g}-\mathrm{m}$ & $3.3 \mathrm{c}-\mathrm{f}$ & 2.3 & S \\
\hline S. chinensis & Rothomagensis & $3.0 \mathrm{~d}-\mathrm{g}$ & $3.2 \mathrm{a}-\mathrm{d}$ & $0.0 \mathrm{~m}$ & 3.7 b-e & 2.5 & $\mathrm{~S}$ \\
\hline S. vulgaris & Alphonse Lavallée & $3.7 \mathrm{~b}-\mathrm{e}$ & $1.8 \mathrm{e}-\mathrm{j}$ & $2.6 \mathrm{a}-\mathrm{f}$ & $3.3 \mathrm{c}-\mathrm{f}$ & 2.6 & $\mathrm{~S}$ \\
\hline S. vulgaris & Krasavitska Moskvy & $2.7 \mathrm{e}-\mathrm{h}$ & $1.8 \mathrm{e}-\mathrm{j}$ & $3.2 \mathrm{a}-\mathrm{c}$ & $3.0 \mathrm{~d}-\mathrm{g}$ & 2.7 & S \\
\hline S. vulgaris & Edmund Boisier & $4.0 \mathrm{a}-\mathrm{d}$ & $2.0 \mathrm{~d}-\mathrm{j}$ & 1.2 g-m & $3.3 \mathrm{c}-\mathrm{f}$ & 2.6 & S \\
\hline S. vulgaris & President Grevy & $2.7 \mathrm{e}-\mathrm{h}$ & $2.4 \mathrm{c}-\mathrm{i}$ & $1.6 \mathrm{e}-\mathrm{k}$ & $4.0 \mathrm{a}-\mathrm{d}$ & 2.7 & $S$ \\
\hline S. vulgaris & Adelaide Dunbar & $4.0 \mathrm{a}-\mathrm{d}$ & $2.2 \mathrm{c}-\mathrm{i}$ & $1.4 \mathrm{f}-1$ & $3.3 \mathrm{c}-\mathrm{f}$ & 2.8 & S \\
\hline S. vulgaris & Charles Joly & $4.3 \mathrm{a}-\mathrm{c}$ & $3.2 \mathrm{a}-\mathrm{d}$ & $1.2 \mathrm{~g}-\mathrm{m}$ & $2.7 \mathrm{e}-\mathrm{h}$ & 2.9 & S \\
\hline S. vulgaris & President Poincare & $3.7 a-b$ & $2.8 \mathrm{~b}-\mathrm{f}$ & $2.6 \mathrm{a}-\mathrm{f}$ & $2.3 \mathrm{f}-\mathrm{i}$ & 2.9 & S \\
\hline S. prestoniae & Minuet & $4.7 a-b$ & $1.2 \mathrm{i}-\mathrm{k}$ & $3.0 \mathrm{a}-\mathrm{d}$ & - - & 3.0 & S \\
\hline S. vulgaris & Montaigne & $4.3 \mathrm{a}-\mathrm{c}$ & $2.2 \mathrm{c}-\mathrm{i}$ & $1.8 \mathrm{~d}-\mathrm{j}$ & 3.7 b-e & 3.0 & S \\
\hline S. vulgaris & Victor Lemoine & $3.0 \mathrm{~d}-\mathrm{g}$ & 3.0 a-e & - & - & 3.0 & S \\
\hline S. vulgaris & Mme. F Morel & 3.7 b-e & $1.6 \mathrm{f}-\mathrm{j}$ & $2.2 \mathrm{~b}-\mathrm{h}$ & $4.3 \mathrm{a}-\mathrm{c}$ & 3.0 & S \\
\hline S. vulgaris & Charles Tenth & $4.7 \mathrm{a}-\mathrm{b}$ & $1.6 \mathrm{f}-\mathrm{j}$ & $2.2 \mathrm{~b}-\mathrm{h}$ & $3.3 \mathrm{c}-\mathrm{f}$ & 3.0 & S \\
\hline S. vulgaris & Mrs. W.E. Marshall & $4.3 \mathrm{a}-\mathrm{c}$ & $1.7 \mathrm{e}-\mathrm{j}$ & - - & $3.3 \mathrm{c}-\mathrm{f}$ & 3.1 & S \\
\hline S. vulgaris & A.M. Brand & $4.7 a-b$ & $2.0 \mathrm{~d}-\mathrm{ij}$ & 3.4 a-b & $2.3 \mathrm{f}-\mathrm{i}$ & 3.1 & S \\
\hline S. vulgaris & Monge & $4.7 a-b$ & $2.6 \mathrm{c}-\mathrm{h}$ & $1.8 \mathrm{~d}-\mathrm{j}$ & $3.3 \mathrm{c}-\mathrm{f}$ & 3.1 & S \\
\hline S. vulgaris & Vestale & $4.7 a-b$ & $3.4 \mathrm{a}-\mathrm{c}$ & $2.4 \mathrm{~b}$ & $2.3 \mathrm{f}-\mathrm{i}$ & 3.2 & S \\
\hline S. vulgaris & Edith Cavell & $5.0 \mathrm{a}$ & 3.00 a-e & $2.6 \mathrm{a}-\mathrm{f}$ & $2.3 \mathrm{f}-\mathrm{i}$ & 3.2 & S \\
\hline S. henryi & White Summers & $4.3 \mathrm{a}-\mathrm{c}$ & $2.2 c-i$ & $3.4 a-b$ & 3.7 b-e & 3.4 & S \\
\hline S. prestoniae & Isabella & $4.7 a-b$ & 3.0 a-e & $2.1 \mathrm{c}-\mathrm{i}$ & 4.0 a-d & 3.4 & S \\
\hline S. josiflexa & Anna Amhof & $3.0 \mathrm{~d}-\mathrm{g}$ & $2.4 \mathrm{c}-\mathrm{i}$ & $3.0 \mathrm{a}-\mathrm{d}$ & $5.0 \mathrm{a}$ & 3.4 & S \\
\hline S. patula & Miss Kim & $4.3 \mathrm{a}-\mathrm{c}$ & $2.6 \mathrm{c}-\mathrm{h}$ & $3.2 \mathrm{a}-\mathrm{c}$ & $3.7 \mathrm{~b}-\mathrm{e}$ & 3.5 & S \\
\hline S. vulgaris & Avalanche & $5.0 \mathrm{a}$ & $2.2 \mathrm{c}-\mathrm{i}$ & $2.6 \mathrm{a}-\mathrm{f}$ & $4.0 \mathrm{a}-\mathrm{d}$ & 3.5 & S \\
\hline S. vulgaris & Arch McKean & $5.0 \mathrm{a}$ & $1.5 \mathrm{f}-\mathrm{j}$ & $3.2 \mathrm{a}-\mathrm{c}$ & $4.7 \mathrm{a}-\mathrm{b}$ & 3.6 & S \\
\hline S. vulgaris & President Lincoln & $5.0 \mathrm{a}$ & $2.6 \mathrm{c}-\mathrm{h}$ & - - & - — & 3.8 & S \\
\hline S. vulgaris & Katherine Havemeyer & $5.0 \mathrm{a}$ & $2.6 \mathrm{c}-\mathrm{h}$ & $3.4 a-b$ & 4.0 a-d & 3.8 & S \\
\hline S. prestoniae & James McFarlane & $5.0 \mathrm{a}$ & $3.2 \mathrm{a}-\mathrm{d}$ & $3.4 a-b$ & - & 3.9 & S \\
\hline S. vulgaris & Mrs. Harry Bickle & $4.7 \mathrm{a}-\mathrm{b}$ & 3.0 a-e & - - & $4.7 \mathrm{a}-\mathrm{b}$ & 4.1 & S \\
\hline S. vulgaris & Marie Finon & $5.0 \mathrm{a}$ & $4.0 \mathrm{a}-\mathrm{b}$ & - - & - - & 4.5 & S \\
\hline S. vulgaris & Marie Legraye & $5.0 \mathrm{a}$ & $4.2 \mathrm{a}$ & - - & - & 4.6 & S \\
\hline $\mathrm{LSD}_{(0.05)}$ & & 1.2 & 1.3 & 0.9 & 1.3 & & \\
\hline
\end{tabular}

${ }^{2}$ Disease readings of $0=$ no symptoms, $1=10 \%, 2=11 \%$ to $25 \%, 3=26 \%$ to $50 \%, 4=51 \%$ to $75 \%$, and $5=76 \%$ to $100 \%$ of the foliage showing disease symptoms. visease reactions categorized as resistant (R), moderately resistant (MR), moderately susceptible (MS), or susceptible (S) were based on the mean disease readings obtained during the growing season in which $\mathrm{R}=0$ to $1.0, \mathrm{MR}=1.1$ to $2.0, \mathrm{MS}=2.1$ to 2.9 , and $\mathrm{S}=3.0$ to 5.0 . Disease readings greater than 2.0 during any growing season disqualified a cultivar from being considered resistant even if the 4-year mean disease reading was less than 2.0; any disease reading of 2.5 to 3.0 during any growing season was an indication of moderate susceptibility, and any reading of greater than 3.0 during a growing season was regarded as fully susceptible. 


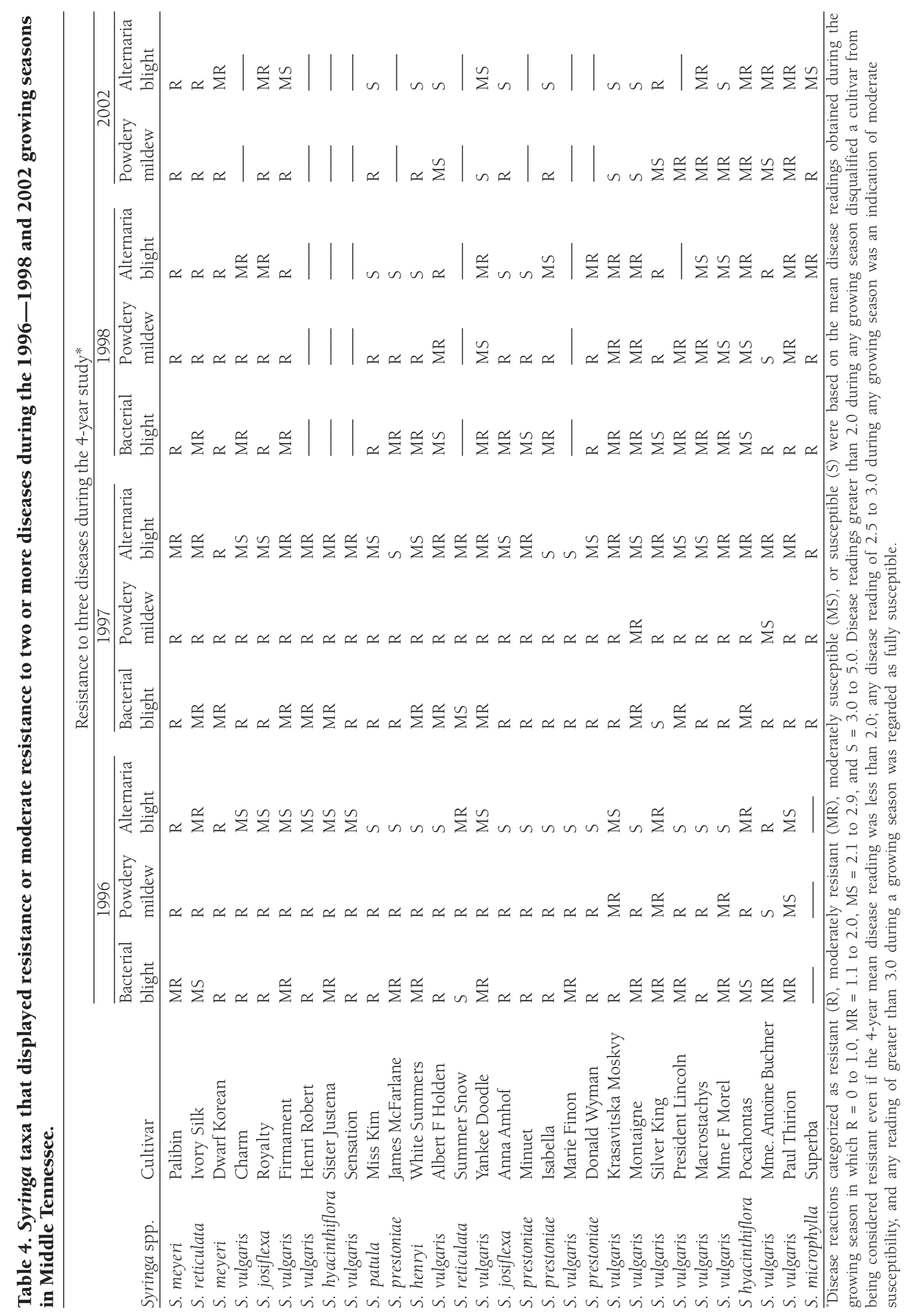

